\title{
Le Rôle DU CONSEIL DE SÉCURITÉ DE L'ORGANISATION DES NATIONS UNIES DANS LES Travaux de la Cour Pénale Internationale
}

\author{
Germana Aguiar Ribeiro do Nascimento* \\ Kahina Merzelkad** \\ María José Añón Roig***
}

\begin{abstract}
1 Introduction. 2 La saisine de la Cour pénale internationale par le Conseil de sécurité : une cooperation en construction. 2.1 La possibilité du Conseil de sécurité de saisir la Cour pénale internationale. 2.2 L'intervention du Conseil de sécurité : vers une plus grande efficacité de la Cour pénale internationale. 3 Le Conseil de sécurité : un obstacle au fonctionnement de la Cour pénale internationale. 3.1 Le pouvoir du Conseil de sécurité d'entraver les activités de la Cour pénale internationale. 3.2 L'influence du Conseil de sécurité dans la détermination de la compétence de la Cour à l'égard des crimes d'agression. 4 Conclusion. Réferences.
\end{abstract}

\section{RESUME}

Le Conseil de sécurité est un organe politique soucieux du maintien de la paix et de la sécurité internationales. La Cour pénale internationale, quant à elle, est un organe juridictionnel subsidiaire qui a pour vocation de juger les crimes de guerre, de génocide, d'agression et les crimes contre l'humanité. Ces deux organes ont comme dénominateur commun le maintien de la paix et de la sécurité internationales. Cette convergence de compétences a été consacrée par le Statut de Rome en octroyant au Conseil de sécurité la prérogative de saisir la Cour. L'intervention du Conseil de sécurité dans le fonctionnement de la Cour peut ainsi être bénéfique pour assurer les droits de l'Homme, néanmoins, elle peut représenter un frein à l'effectivité de l'exercice de la compétence

* Bacharela em Direito, pela Universidade Estadual da Paraíba. Bacharela em Antropologia, pela Universidade Federal de Campina Grande. Mestre em Direito Internacional dos Direitos Humanos, pela Universidade Católica de Lyon - França. Doutoranda em Direitos Humanos, Democracia e Justiça Internacional, pela Universidade de Valência - Espanha. E-mail: <germana_aguiar@hotmail.fr>.

** Doutoranda em Direito Internacional da Université Pierre-Mendès France - Grenoble II. <merzelkahina@yahoo.fr>.

*** Professora efetiva de Filosofia do Direito da Universitat de València - Espanha. Licenciada e Doutora em Direito pela Universitat de València - Espanha. Diretora do Instituto Universitario de Derechos Humanos (2005-2010). Secretaria Geral da Universitat de València (2010 - 2016). Ex-diretora do Anuario de Filosofía del Derecho e atual diretora da Teoría y derecho. Revista de pensamiento jurídico.<maria.j.anon@uv.es>. 
de la Cour. Dans cette optique, il serait intéressant d'aborder l'aspect constructif du lien qui unit la Cour et le Conseil de sécurité, ainsi que l'obstacle qu'il peut représenter pour la réalisation de la paix et de la sécurité internationales. Pour cela, la méthodologie qualitative sera utilisée. La recherche documentaire, l'étude de la jurisprudence et de la législation permettront de regrouper les renseignements relatifs au sujet.

Mots-clés: Conseil de sécurité. Cour pénale internationale. Droits de l'Homme.

\section{INTRODUCTION}

Il appartiendrait à la Cour d'agir de manière rapide, indépendante et impartiale, à la recherche d'un difficile point d'équilibre entre les exigences de la justice et celles de la politique, de la paix et de la sécurité internationales. ${ }^{1}$ Cette affirmation démontre les difficultés que rencontre la Cour pénale internationale pour établir une relation harmonieuse avec les autres organes onusiens, particulièrement avec le Conseil de sécurité. La clef de voûte de cet équilibre peut être la convergence des objectifs de chacune de ces institutions, ayant pour postulat la réalisation de la paix et de la justice.

En effet, on peut constater que dans l'histoire de l'humanité, la recherche de l'effectivité de ces objectifs a nécessité la mise en œuvre de mécanismes de répression et de sanctions. Après la Seconde Guerre Mondiale, la communauté internationale prend conscience de la gravité des dégâts générés par les conflits et du mépris des libertés et des droits de l'Homme. ${ }^{2}$ Cela suscite la création des mécanismes juridictionnels qui ont pour prétention d'assurer la justice et la paix dans le monde.

Le Tribunal militaire de Nuremberg, créé en 1945 pour juger les grands criminels du Troisième Reich, représente un de ces premiers instruments juridictionnels. Cependant, malgré la bonne volonté des Alliés à réprimer ces crimes, plusieurs critiques vont apparaitre concernant la partialité de ce dernier, car il s'agissait d'un tribunal de vainqueurs contre des vaincus. Hors, pour que la justice règne, le vaincu comme le vainqueur doit répondre des atrocités dont il a été l'auteur, si les preuves sont apportés. ${ }^{3}$ Le Tribunal de Nuremberg dénotait ainsi un aspect inéquitable de la procédure.

Ce n'est qu'à travers l'initiative du Conseil de sécurité de créer le Tribunal pénal international pour l'ex-Yougoslavie en 1993 et le Tribunal pénal international pour le Rwanda en 1994, que la justice internationale va réellement jouer un rôle primordial dans la répression des crimes les plus graves contre l'humanité. En effet, il faut attendre la fin de la GuerrefFroide pour voir renaître l'espoir d'une justice pénale internationale effective. ${ }^{4}$ C'est à partir de ces expériences que la communauté internationale va repenser les possibilités de répression de ces crimes, en créant une Cour pénale internationale en 1998, par le biais d'un traité multilatéral, le Statut de Rome. 
Ce nouvel organe juridictionnel avait pour objectif l'instauration d'une Cour Permanente afin de juger telles atrocités, et d'éviter dorénavant la création par le Conseil de sécurité de nouveaux tribunaux ad hoc.

En effet, ce Statut affirme que la Cour a la compétence de juger les crimes de guerre, de génocide, d'agression et les crimes contre l'humanité. Cette compétence rationae materiae se rapproche de celle prévue par le Chapitre VII de la Charte des Nations Unies. Ce Chapitre définit les prérogatives du Conseil de sécurité dans le cadre des crimes qui menacent le maintien de la paix et de la sécurité internationales. Cette concomitance de compétences va permettre au Conseil de sécurité de saisir la Cour.

Toutefois, cette faculté accordée au Conseil de sécurité fait l'objet de plusieurs polémiques. En effet, malgré la présence d'objectifs similaires, l'activité de chacun de ces organes peut être incompatible, en vertu du caractère politique de l'un et du caractère juridictionnel de l'autre. Ce qui montre la difficulté de la Cour de trouver un point d'équilibre, tout en maintenant son indépendance et sa crédibilité. Il s'agit ainsi, d'analyser de quelle manière le Conseil de sécurité contribue aux travaux de la Cour pénale internationale.

Il est vrai que l'intervention du Conseil de sécurité dans le fonctionnement de la Cour peut être bénéfique pour assurer le maintien de la paix et de la sécurité internationales, néanmoins, elle peut représenter un frein à l'effectivité de l'exercice de la compétence de la Cour. Dans cette optique, il serait intéressant d'aborder l'aspect constructif du lien qui unit la Cour et le Conseil de sécurité, ainsi que l'obstacle qu'il peut représenter.

\section{LA SAISINE DE LA COUR PÉNALE INTERNATIONALE PAR LE CONSEIL DE SÉCURITÉ : UNE COOPERATION EN CONSTRUCTION}

Le préambule du Statut de Rome affirme que le but de la Cour pénale internationale est la promotion de la justice internationale, à travers la répression des crimes les plus graves. Le Conseil de sécurité, quant à lui, a comme objectif, ce qui est défini dans l'article 24 (1) de la Charte des Nations Unis, le maintien de la paix et de la sécurité internationales. Dans ce sens, il est possible d'établir un lien entre ces deux organes, vu qu'ils ont a priori des objectifs convergents. Telle affirmation est corroborée par le Statut de la Cour, qui prévoit la possibilité du Conseil de sécurité de la saisir, ce qui représenterait un aspect bénéfique pour son fonctionnement.

2.1 La possibilite du conseil de securite de saisir la cour penale internationale

La prévision de la saisine de la Cour pénale internationale par le Conseil de sécurité est la conséquence du développement des mécanismes de répression de crimes contre l'humanité. En effet, le Conseil de sécurité a créé, à travers la Résolution 827 du 25 mai 1993, le Tribunal pénal international pour l'ex-Yougoslavie et, à travers la Résolution 955 du 8 novembre 1994, le Tribunal 
pénal international pour le Rwanda. Tels tribunaux ad hoc ont généré plusieurs querelles entre les Etats.

En effet, plusieurs d'entre eux ont critiqué cette création en contestant la compétence du Conseil de sécurité, en soulignant les risques d'une justice inéquitable, les frais de la procédure et la difficulté de sa mise en place. Ainsi, la création de la Cour pénale internationale et la possibilité du Conseil de la saisir viennent dans le sens d'éviter les situations mentionnées, qui n'étaient plus souhaitables. ${ }^{5}$

Il est impératif d'affirmer que le seul organe des Nations Unies qui peut saisir la Cour pénale internationale est le Conseil de sécurité. Toutefois, le premier projet du Statut de la Cour, élaboré en 1951 par un Comité composé de dix-sept Etats membres, donnait cette faculté aussi à l'Assemblée générale, ${ }^{6}$ en vertu de sa responsabilité de protéger les droits de l'Homme et de coopérer dans le maintien de la paix et de la sécurité. ${ }^{?}$

Telle faculté était liée au contexte de la Guerre froide, quand les activités du Conseil de sécurité étaient bloquées par les vetos des Etats-Unis et de l'ex-URSS. En effet, comme affirme Barcelo ${ }^{8}$ :

La guerre froide a marqué un coup d'arrêt aux discussions engagées sur un rythme soutenu dans la perspective de créer une juridiction pénale internationale. Elle a offert aux principales puissances de la planète un prétexte substantiel pour éviter une discussion de fond qui remettrait en cause leur souveraineté.

Avec la fin de la Guerre froide, les acteurs du Conseil de sécurité se sont intégrés et devenus plus forts. Au-delà de cela, les recommandations faites par l'Assemblée générale n'ont pas un caractère obligatoire, au contraire de celles faites par le Conseil de sécurité. Ainsi, le projet n'a pas abouti, et seulement le Conseil de sécurité, en tant qu'organe des Nations Unies, a la possibilité de saisir la Cour.

Cette faculté est envisagée dans l'article 13 du Statut, qui affirme que

La Cour peut exercer sa compétence à l'égard des crimes visés à l'article 5, conformément aux dispositions du présent Statut: [...] b) Si une situation dans laquelle un ou plusieurs de ces crimes paraissent avoir été commis est déférée au Procureur par le Conseil de sécurité agissant en vertu du chapitre VII de la Charte des Nations Unies;

Cet article précise ainsi dans quels cas le Conseil de sécurité a l'autorité de saisir la Cour. Cependant, son contenu dénote certaines lacunes qui peuvent faire l'objet de discussion.

Tout d'abord, il s'agit d'analyser quelles sont les modalités de la saisine par le Conseil, puisqu'aucun article ne décrit cela. Différemment de l'article 16, ${ }^{9}$ qui impose la nécessité d'une résolution formelle pour suspendre les poursuites et les enquêtes, l'article 13 affirme seulement la nécessité d'une situation "éférée au 
Procureur». Selon Dulait, ${ }^{10}$ plusieurs indices démontrent qu'une décision doit être exigée, ce qui est corroborée par les travaux préparatoires du Statut. Ainsi, selon l'article 39 de la Charte des Nations Unies, premièrement, le Conseil va constater l'existence d'une menace contre la paix, une rupture de la paix ou un acte d'agression, dans une situation de crime soumis à la compétence de la Cour. Après cela, le Conseil peut décider quelle mesure il va prendre, dans le cadre de l'article $41,{ }^{11}$ en pouvant saisir la Cour.

Selon l'article 13 du Statut, il est possible de saisir la Cour par trois moyens. Elle peut être saisie par un Etat parti; par le Procureur lui-même et par le Conseil de sécurité. Cependant, la troisième possibilité semble être la plus efficace. En effet, elle n'est pas soumise à la condition de consentement préalable des Etats à la compétence de la Cour, comme affirme l'article 12. Cet article impose cette condition seulement aux alinéas (a) et (c) de l'article 13. De plus, l'article 25 de la Charte des Nations Unies dispose que : "Les membres de l'Organisation conviennent d'accepter et d'appliquer les décisions du Conseil de sécurité conformément à la présente Charte", ce qui corrobore l'inexistence de la nécessité d'un consentement préalable quand le Conseil prendre une décision selon le Chapitre VII. Dans ce sens, les Etats non partis du Statut de la Cour peuvent être soumis à sa compétence par le Conseil de sécurité et doivent être coopératifs. Telle possibilité a été critiquée par l'Inde, qui n'a pas voulu signer le Statut. Cependant, la position de cet Etat ne remet pas en cause, pour autant, l'aspect positif de l'extension de la compétence de la Cour, puisqu'elle exclurait les risques d'impunité. ${ }^{12}$

Ainsi, le Conseil est soumis, lorsqu'il saisit la Cour à tous les articles du Statut. Il ne peut pas la saisir hors du Chapitre VII de la Charte, ni utiliser un régime différent de celui prévu. De plus, il doit respecter l'article 11, qui affirme que : "La Cour n'a compétence qu'à l'égard des crimes relevant de sa compétence commis après l'entrée en vigueur du présent Statut (...)”. Il s'agit de la compétence rationae temporis, par conséquent, les crimes commis avant l'entrée en vigueur du Statut ne peuvent pas subir le jugement de la Cour. Il doit encore respecter les compétences prévues par les articles 5 et 25, respectivement, rationae materiae et ratione personae. Selon l'article 53, le Procureur peut décider de ne pas poursuivre la demande du Conseil de sécurité. Le Conseil, à son tour, peut demander le réexamen de la question.

Malgré le caractère d'universalité, la réalité démontre que la saisine de la Cour par le Conseil de sécurité va dépendre plusieurs fois d'une volonté politique de ses membres, ce qui crée un obstacle pour l'effectivité de tel instrument. Par exemple, l'inaction du Conseil de sécurité est constatée par rapport au Timor-Leste, ce qui est dénoncée par Amnesty International, ${ }^{13}$ qui accuse le Conseil de sécurité de repousser l'examen d'un rapport détaillé sur la violation grave des droits de l'Homme dans ce pays. Toutefois, il est possible d'affirmer que l'intervention du Conseil de sécurité peut être bénéfique pour le fonctionnement de la Cour pénale internationale. 
2.2 L'intervention du conseil de securite: vers une plus grande efficacite de la cour penale internationale

Le rôle du Conseil de sécurité dans le fonctionnement de la Cour pénale internationale peut sembler positif, puisqu'il est capable d'aller au delà des attributions du principe de la complémentarité. En effet, le préambule du Statut reconnaît un rôle premier de chaque Etat dans la répression des crimes internationaux. Dans le même sans, il affirme dans son article premier, que la Cour pénale internationale sera complémentaire des juridictions criminelles nationales. Ce qui peut être expliqué par l'envie des Etats de préserver leur souveraineté et de laisser à la Cour une tâche secondaire dans la répression des crimes.

Ainsi, le recours au principe de la subsidiarité doit permettre à la CPI d'avoir une action efficace tout en respectant les souverainetés nationales. Cependant, dans l'article 17, il est affirmé que la Cour peut recevoir une affaire, quand l'Etat est incapable ou s'abstient de réprimer les crimes prévus par l'article 5. Ainsi, le Statut essaye d'établir un équilibre entre les compétences des Etats et celle de la Cour, pour que la justice soit garantie, même si l'Etat est défaillant dans sa justice nationale.

En effet, l'objectif initial de l'installation de la Cour était ${ }^{14}$

mettre en place une institution permanente permettant d'éviter la création d'un tribunal spécial à chaque fois qu'un génocide, des crimes de guerre ou des crimes contre l'humanité seraient commis et que les juridictions nationales compétentes ne fonctionneraient pas comme elles le devraient. Malheureusement, l'histoire démontre clairement que les systèmes nationaux se trouvent dans l'incapacité de tenir leur rôle lorsque les crimes les plus graves sont commis ou que la volonté de les juger fait défaut. Il suffit d'évoquer l'Allemagne nazie, le Cambodge, l'Ouganda d'Idi Amin Dada, le Rwanda ou l'ex-Yougoslavie pour illustrer ce constat.

L'article 17 du Statut détermine ainsi quelles sont les circonstances qui démontrent la défaillance de l'Etat, telle que le retard injustifié de la procédure ou le non respect des normes d'indépendance et d'impartialité par les juridictions nationales. Ce principe est applicable aussi à la saisine émanée du Conseil de sécurité, mais d'une façon différente. En effet, l'article 18 (1) du Statut affirme que:

Lorsqu'une situation a été déférée à la Cour comme le prévoit l'article 13, alinéa a), et que le Procureur a déterminé qu'il y aurait une base raisonnable pour ouvrir une enquête, ou lorsque le Procureur a ouvert une enquête au titre des articles 13, paragraphe c), et 15, le Procureur le notifie à tous les États Parties et aux États qui, selon les renseignements disponibles, auraient normalement compétence à l'égard des crimes dont il s'agit. 
Il est possible d'observer que cet article ne cite pas la saisine émanée du Conseil de sécurité, ce qui dénote une lacune qui permet à ce dernier d'agir au delà des limites posées par le Statut de Rome. Cette option octroyée à cet organe peut garantir, d'un certain point de vue, une plus grande efficacité dans la gestion de certains conflits, en les déferant directement à la Cour. Cette plus grande efficacité est aussi remarquée dans le cadre de la non coopération des Etats.

Pour l'efficacité de la Cour, la coopération des Etats est indispensable, puisqu'elle ne possède pas d'une force de police ${ }^{15}$. Telle coopération est définie dans le Chapitre IX, qui définit les modalités de coopération internationale et de l'assistance judiciaire. Cependant, ces dispositions autorisent au même temps les Etats de s'abstenir de cette obligation.

En effet, l'article 94 qui permet la possibilité d'un plus grand délai pour l'exécution d'un acte de coopération ou l'article 89 qui prévoit l'utilisation des législations nationales dans certaines procédures, créent des difficultés pour l'efficacité de la Cour. Toutefois, de telles difficultés peuvent être contournées quand la saisine émane du Conseil de sécurité. Ainsi, quand le Conseil de sécurité saisit la Cour, tous les Etats membres des Nations Unies sont obligés de coopérer avec elle, même ceux qui ne font pas partie du Statut de Rome, comme affirme l'article 25 de la Charte des Nations Unies, ce qui va bénéficier le fonctionnement de la Cour.

\section{De plus, l'article 87 (7) du Statut dispose que}

Si un État Partie n'accède pas à une demande de coopération de la Cour contrairement à ce que prévoit le présent Statut, et l'empêche ainsi d'exercer les fonctions et les pouvoirs que lui confère le présent Statut, la Cour peut en prendre acte et en référer à l'Assemblée des États Parties ou au Conseil de sécurité lorsque c'est celui-ci qui l'a saisie.

Toutefois, l'Assemblée des Etats Parties ne peut pas infliger, au contraire du Conseil de sécurité, des sanctions efficaces aux Etats qui refusent de coopérer avec la Cour. En effet, il n'y a aucune prévision dans ce sens. Le Conseil, quant à lui, peut garantir ces sanctions, quand c'est lui qui saisit la Cour, puisqu'elles sont prévues dans les articles 41 et 42 du Statut. Cependant, il pourra agir seulement quand il y a une menace au maintien de la paix et de la sécurité internationales, comme l'affirme l'article 24 de la Charte. Dans ce sens, il ne peut pas établir des sanctions seulement pour garantir l'obligation de coopérer.

Dans le cas de la Libye, par exemple, le Conseil de sécurité a déterminé des mesures des sanctions, conformément à la Résolution 1973, ${ }^{16}$ qui impose l'embargo sur les armes, du gel des avoirs des autorités de Tripoli et l'interdiction de vol des compagnies aériennes libyennes. Dans ce sens

Tous les Etats membres doivent prendre immédiatement les mesures nécessaires pour empêcher la fourniture, la vente ou le transfert directs ou indirects à la Jamahiriya libyenne, à partir de 
leur territoire ou par leurs nationaux, ou au moyen de navires ou d'aéronefs battant leur pavillon, d'armements et de matériel connexe de tous types.

Cependant, le Conseil de sécurité, en pratique, n'a pas toujours exercé son pouvoir de sanction, tel qu'au Kosovo, en 1998, quand il a condamné la République Fédérative d'Yougoslavie, sans établir aucune sanction.

Ainsi, il apparaît que l'intervention du Conseil de sécurité dans les travaux de la Cour peut tendre vers une coopération constructive. Le procès de l'ancien chef d'Etat ivoirien, Laurent Gbagbo, devant la Cour pénale internationale, qui a débuté le 28 janvier 2016, est une illustration parfaite du rôle important que peuvent représenter ces deux organes en matière de maintien de la paix et de la sécurité internationales. Cependant, les intérêts poursuivis par le Conseil en tant qu'organe politique peuvent facilement entraver le bon fonctionnement de la Cour pénale internationale.

\section{LE CONSEIL DE SÉCURITÉ: UN OBSTACLE AU FONCTIONNE- MENT DE LA COUR PÉNALE INTERNATIONALE}

La compréhension de la relation particulière qui lie la Cour pénale internationale au Conseil de sécurité est la pierre angulaire qui permet de mieux cerner la question de l'effectivité de la justice internationale. ${ }^{17}$ En effet, le préambule du Statut de Rome est très significatif à ce sujet en affirmant sa détermination de "créer une Cour pénale internationale permanente et indépendante reliée au système des Nations Unies". Cette affirmation est renforcée par les dispositions du Statut qui reconnaissent au Conseil de sécurité un rôle spécifique dans le fonctionnement de la Cour. Cependant, ce rôle a souvent été contesté car il lui octroi la possibilité d'interférer dans les activités de cette dernière et de limiter sa compétence à l'égard des crimes d'agression.

3.1 Le pouvoir du conseil de securite d'entraver les activites de la cour penale internationale

Les dispositions du Statut de Rome attribuent au Conseil de sécurité des facultés particulières lui accordant le pouvoir de déférer à la Cour des situations qui menacent le maintien de la paix et la possibilité de surseoir les enquêtes de cette dernière.

L'article 13(b) du Statut affirme que le Conseil de sécurité est l'autorité qui a la faculté de déférer à la Cour une situation caractérisée comme crime selon l'article $5 \mathrm{du}$ Statut. Cet article est confirmé par l'article 17 de l'accord régissant les relations entre la Cour pénale et les Organisations des Nations Unies signé le 4 octobre 2004 en disposant que "le secrétaire général transmet immédiatement la décision écrite du Conseil de sécurité au Procureur avec les documents et autres pièces pouvant s'y rapporter" et que la Cour s'engage à tenir le Conseil de sécurité informé. ${ }^{18}$ 
Ces pouvoirs octroyés au Conseil soulèvent quelques ambigüités quant au principe d'égalité entre les Etats. En effet, l'article 34 de la Convention de Vienne sur le droit des traités du 23 mai 1969 dispose qu' : «Un traité ne crée ni obligations ni droits pour un Etat tiers sans son consentement». Cet article implique le respect de la volonté des Etats à adhérer ou non à un traité. Ainsi, Kirsch $^{19}$ affirme que la "CPI n'a pas la compétence universelle, elle ne peut exercer sa compétence qu'avec le consentement de l'Etat du territoire où le crime a été commis ou de l'Etat de la nationalité de l'accusé". Cependant, le pouvoir d'initiative du Conseil provoque une extension du domaine subjectif du Statut aux Etats qui n'ont pas adhéré au traité, ce qui peut remettre en question le principe du fonctionnement consensuel et le principe pacta tertiis nec nocent nec prosunt.

Cette entorse au principe de l'égalité entre les Etats est aussi décelable concernant la procédure de véto et la protection qu'elle procure aux membres permanents du Conseil. Ces derniers peuvent voter une résolution pour déférer un Etat devant la Cour pénale, alors qu'eux-mêmes ne sont pas partis au Statut de Rome. ${ }^{20}$ La reconnaissance de cette immunité a été réaffirmée par le Conseil de sécurité dans la résolution 1422 adopté le 12 juillet 2002, selon laquelle le Conseil s'engage à ne mener aucune enquête ou poursuite ${ }^{21}$ à l'encontre des ressortissants des Etats contributeurs aux opérations de maintien de la paix. C'est ainsi que les Etats Unis et ses citoyens seraient à l'abri de toute poursuite qui remettrait en cause leur politique étrangère. ${ }^{22}$

Ainsi, il apparaît que cette possibilité d'intervention conférée au Conseil de sécurité représente une entrave pour l'effectivité de la compétence de la Cour, qui se poursuit avec la faculté du Conseil de bloquer toute enquête et toute poursuite engagées par cette dernière. En effet, l'article 16 du Statut de Rome dispose que

Aucune enquête ni aucune poursuite ne peuvent être engagées ni menées en vertu du présent Statut pendant les douze mois qui suivent la date à laquelle le Conseil de sécurité a fait une demande en ce sens à la Cour dans une résolution adoptée en vertu du Chapitre VII de la Charte des Nations Unies; la demande peut être renouvelée par le Conseil dans les mêmes conditions.

En d'autres termes, cet article octroi au Conseil la possibilité de mettre en attente pendant douze mois les enquêtes et les poursuites de la Cour pénale pour permettre la mise en œuvre d'un accord de paix.

En partant de cette hypothèse, le Conseil doit reconnaitre dans la demande qu'il adresse à la Cour l'existence d'une menace à la paix, d'une rupture de la paix ou d'un acte d'agression conformément à l'article 39 de la Charte. Or, les deux seules résolutions 1422 (2002) et 1487 (2003) adoptées par le Conseil de sécurité sur la base de l'article 16 du Statut de Rome, ne faisaient à aucun moment référence à une quelconque menace au maintien de la paix mais se limitaient à attribuer aux ressortissants des Etats contributeurs aux opérations 
de maintien de la paix l'immunité pendant la période établie par l'article susmentionné. Ce qui laisse apparaître une extension du caractère politique du Conseil sur le caractère juridictionnel de la Cour. ${ }^{23}$ Cette limitation temporelle de la compétence de la Cour en faveur de l'impérial maintien de la paix risquerait ainsi de porter atteinte au pouvoir dissuasif de cette dernière à l'encontre des violations des droits de l'Homme ${ }^{24}$ et crée une forme de subordination de la Cour par rapport au Conseil.

\section{Pour certains spécialistes, tel que Poitevi ${ }^{25}$}

les demandes de sursis à enquêter du Conseil de sécurité devraient faire l'objet d'un contrôle de légalité par la Cour. Bien qu'il ne soit pas explicitement prévu par le Statut, il pourrait se baser sur l'article 19 § 1 disposant que "la Cour s'assure qu'elle est compétente pour connaître de toute affaire portée devant elle», autrement dit que la Cour dispose de la «compétence de la compétence.

Cette proposition semble très pertinente en imposant au Conseil d'établir le lien de causalité entre la menace du maintien de la paix et la demande de sursis à enquêter. Ce qui renforcerait la légitimité de la Cour pénale en tant qu'organe compétent en matière de justice internationale et non assujetti au conseil. Cette légitimité de la compétence de la Cour gagnerait aussi à être renforcée à l'égard des crimes d'agression.

3.2 L'influence du conseil de securite dans la determination de la competence de la cour a l'egard des crimes d'agression

Le crime d'agression fait partie des crimes les plus graves, qui touchent l'ensemble de la communauté internationale et relèvent de la compétence de la Cour pénale. L'inclusion de ce crime dans l'article 5 a suscité plusieurs discussions. En effet, l'absence de la définition de ce crime rendait la compétence de la Cour illusoire, jusqu'à l'adoption des amendements de révision du Statut, qui ont déterminé les conditions de sa mise en pratique. Néanmoins, ces dispositions attribuent un rôle privilégié et discutable au Conseil de sécurité.

L'article 5 du Statut de Rome dresse une liste des crimes graves qui relèvent de la compétence de la Cour notamment le crime d'agression. Cependant l'alinéa 2 de ce même article dispose que

La Cour exercera sa compétence à l'égard du crime d'agression quand une disposition aura été adoptée conformément aux articles 121 et 123, qui définira ce crime et fixera les conditions de l'exercice de la compétence de la Cour à son égard. Cette disposition devra être compatible avec les dispositions pertinentes de la Charte des Nations Unies.

La formulation de cette norme suspend ainsi la compétence de la Cour tant qu'une définition du crime d'agression ne sera pas adoptée par le biais d'amendements au terme des articles 121 et 123 du Statut. Ces derniers 
soumettent l'adoption des révisions à des procédures particulières telles que l'expiration de la période de sept ans à compter de la date de l'entrée en vigueur du Statut, ce qui dénote un caractère fictif de la compétence de la Cour. De plus le dernier paragraphe de l'article 5 du Statut de Rome rappelle le rôle du Conseil de sécurité et l'obligation de respecter les dispositions de la Charte des Nations Unies. Les amendements ainsi adoptés devront assurer la responsabilité primaire du Conseil de maintenir la paix et la sécurité dans le monde au détriment de la compétence de la Cour si cela s'avère nécessaire.

Ainsi, sur la base de l'article 5 du Statut et de la réflexion susmentionnés, des amendements ont été adoptés lors de la Conférence de révision du Statut de Rome en 2010 afin de donner une définition au crime d'agression, de déterminer les conditions d'exercice de la compétence de la Cour et de délimiter par la même occasion le rôle du Conseil de sécurité.

Le 11 juin 2010 lors de la Conférence de révision du Statut de Rome à Kampala en Ouganda, les Etats parties aux discussions ont adopté par consensus des amendements qui portaient essentiellement sur la définition du crime d'agression et sur les conditions de saisine de la Cour pénale.

La définition retenue par les Etats reprend pour l'essentiel les termes de l'agression contenue dans la résolution 3314 (XXIX) de l'Assemblée générale des Nations Unies du 14 décembre 1974. En effet, l'article article 8 bis, paragraphe 1 , dispose que le crime d'agression sous-entend

la planification, la préparation, le lancement ou l'exécution par une personne effectivement en mesure de contrôler ou de diriger l'action politique ou militaire d'un État, d'un acte d'agression qui, par sa nature, sa gravité et son ampleur, constitue une violation manifeste de la Charte des Nations Unies.

Le paragraphe 2 de cet article définit aussi l'acte d'agression comme étant "l'emploi par un État de la force armée contre la souveraineté, l'intégrité territoriale ou l'indépendance politique d'un autre État, ou de toute autre manière incompatible avec la Charte des Nations Unies".

La formulation de cette définition s'appuie largement sur les termes et les dispositions du Statut de Rome et de la Charte de Nations Unies et reflètent un compromis trouvé par de nombreux États avant la Conférence de révision. ${ }^{26}$ Ce qui permet à la Cour désormais d'exercer théoriquement sa compétence à l'égard des crimes d'agression sous certaines conditions.

L'article 15 bis, paragraphe 6 , des amendements relatifs au crime d'agression dispose que:

La Cour peut exercer sa compétence à l'égard du crime d'agression conformément aux paragraphes a) et c) de l'article 13, sous réserve des dispositions qui suivent.(...) 6 - Lorsque le Procureur conclut qu'il y a de bonnes raisons de mener une enquête pour crime d'agression, il s'assure d'abord que le Conseil de sécurité 
a constaté qu'un acte d'agression avait été commis par l'État en cause. Il avise le Secrétaire général de l'Organisation des Nations Unies de la procédure judiciaire engagée devant la Cour et lui communique toute information et tout document utile.

Ce paragraphe sous-entend que le Procureur ne peut entamer une enquête de sa propre initiative que s'il a vérifié que le crime d'agression a bien été constaté par le Conseil de sécurité. Ce qui maintient la Cour suspendue à l'action ou l'inaction de ce dernier.

Le paragraphe 7 de l'article susmentionné semble donner une solution transitoire en précisant que si une telle constatation n'est pas faite par le Conseil de sécurité dans le délai de six mois suivant la date de l'avis, le Procureur peut mener une enquête de son propre chef. Toutefois, cette permission accordée à la Cour pénale est soumise à certaines conditions. En effet, le Procureur doit obtenir l'accord de la chambre préliminaire et s'assurer que le Conseil n'a pas décidé de surseoir son enquête conformément à l'article 16 du Statut.

Ainsi, il apparaît que la capacité de la Cour à enquêter reste subordonnée à la décision du Conseil de sécurité. Ce dernier demeure le principal détenteur de la mission de qualifier un acte d'agression. Ce qui semble poser la question de l'indépendance de la Cour pénale à l'égard du Conseil de sécurité, qui est avant tout un organe politique qui obéit aux intérêts des Etats parties en particulier les membres permanents.

Il faut aussi noter, que l'exercice de la compétence de la Cour à l'égard des crimes d'agression ne pourra s'activer qu'à partir du 1er janvier 2017 suite à la décision prise par la majorité des Etats requise pour l'adoption d'un amendement au Statut. De plus, tout Etat partie sera libre de déclarer ne pas reconnaître la compétence de la Cour pour le crime d'agression en déposant une déclaration en ce sens auprès du Greffier de la cour selon l'article 15 bis chapitre 4 de l'amendement susmentionné. Ce qui au final permet aux responsables de crime internationaux des Etats non parties aux amendements notamment certains membres permanents du Conseil de sécurité (les Etats Unis), d'échapper à la compétence de la Cour pénale.

Ainsi il apparaît que cette révision du Statut de Rome a certes contribué à la définition du crime d'agression et de ses éléments constitutifs, ce qui n'en est pas de même pour l'exercice de la compétence de la Cour. Cet organe juridictionnel reste toujours de manière implicite subordonné à l'autorité du Conseil de sécurité ce qui pourrait ouvrir la porte à une forme de politisation de la Cour pénale internationale et l'éloignerait de ses objectifs principaux, à savoir, une meilleure mise en œuvre du Droit international humanitaire, la lutte contre l'impunité et la promotion de la justice. Comme l'affirme Sur "on ne peut totalement dissocier justice et maintien de la paix, dans la mesure où l'action publique, même judiciaire, a pour ultime ressort la paix publique». ${ }^{27}$ 


\section{CONCLUSION}

Le Conseil de sécurité est un organe politique soucieux du maintien de la paix et de la sécurité internationales. Quant à la Cour pénale, elle est un organe juridictionnel subsidiaire qui a pour vocation de juger les crimes de guerre, de génocide, d'agression et les crimes contre l'humanité. Ces deux organes ont comme dénominateur commun le maintien de la paix et de la sécurité internationales. Cette convergence de compétences a d'ailleurs été consacrée par le Statut de Rome en octroyant au Conseil de sécurité la prérogative de saisir la Cour. Cette consécration offre une plus grande efficacité de la Cour à travers une extension de sa compétence et la possibilité de sanction que peut exercer le Conseil de sécurité sur les Etats, lorsque le maintien de la paix et de la sécurité internationales sont menacées.

Cependant, le caractère politique du Conseil de sécurité peut représenter un obstacle au bon fonctionnement de la Cour pénale internationale. Le pouvoir du Conseil de sécurité de déférer un Etat n'étant pas partie au Statut de Rome devant la Cour et la réduction de la compétence de cette juridiction internationale à l'égard des crimes d'agression sont les principales critiques émises vis-à-vis de cette combinaison politico-juridictionnelle.

Ainsi, la Cour pénale internationale reste implicitement assujettie au pouvoir du Conseil de sécurité. L'efficacité de cet organe juridictionnel dépend de sa totale indépendance et nécessité un éloignement de la sphère politique. Ce vœu pieux est difficilement réalisable compte-tenu des prérogatives accordées par la Chartes des Nations Unies aux membres permanents du Conseil de sécurité et à la forte influence que peuvent représenter les intérêts politico-économiques. La Cour pénale internationale reste tout de même un organe juridictionnel important pour juger les atrocités humaines et lutter contre l'impunité qu'il est impératif de préserver.

\section{REFERENCES}

AMNESTY INTERNATIONAL. Bulletin d'information 228/2005. 2005. Disponible en: <http://www.amnesty.org/fr/library/asset/ASA57/004/2005/ fr/62a24ca6-d4b7-11dd-8a23-d58a49c0d652/asa570042005fr.html>. Accès le : 15 février 2016.

BARCELO, Laurent. Aux origines de la Cour pénale internationale: le projet français de chambre criminelle internationale (hiver 1946 - printemps 1947). Guerres mondiales et conflits contemporains, France, n. 222, p. 103-109, 2006/2.

BOURGUIBA, Leïla. Cour pénale internationale, modèles de saisine et limites. Confluence méditerranée, justice pénale et politique internationale, Paris, n. 64, p. 198, hiver 2007-2008. 
CASSESSE, Antonio. Los derechos humanos em el mundo contemporaneo. Barcelona: Ariel,1993.

CENTRE D'ACTUALITES DE L'ONU. Libye: le Conseil de sécurité adopte des sanctions. Disponible en: $<$ http://www.un.org/apps/newsFr/storyF.as$\mathrm{p} ? \mathrm{NewsID}=24578 \& \mathrm{Cr}=\mathrm{Libye} \& \overline{\mathrm{Cr}} 1 \geq$. Accès le $: 20$ février 2016.

ÇEHRELI, Ayse Sila. L'odyssée du XXe siècle: la naissance de la Cour pénale internationale. Synergies Turquie, France, n 2, p. 113-122, 2009.

CHIAVARIO, Mario. La justice pénale internationale entre passé et avenir. Paris: Editions DALLOZ, 2003.

CONSEIL DE SECURITE. Résolution 955 du 8 novembre 1994. S/RES/955. Disponible en: <http://www.francerwandagenocide.org/documents/93s827. pdf $\geq$. Accès le: $\overline{15}$ février 2016.

CONSEIL DE SECURITE. Résolution 827 du 25 mai 1993. S/RES/827. Disponible en: <http://www.francerwandagenocide.org/documents/93s827. pdf $>$. Accès le: $\overline{15}$ février 2016.

DULAIT, André. Rapport sur Le rôle du Conseil de sécurité sur le fonctionnement de la Cour pénale internationale. 1999. Disponible en: $\leq$ http://www. senat.fr/rap/r98-313/r98-3139.html>. Accès le: 15 février 2016.

FOFE DJOVIA MALEWA, Jean-Pierre. La Cour pénale internationale: institution nécessaire aux pays des grands lacs africains. Paris: L'Harmattan, 2006.

FROUVILLE, Olivier de. Droit International pénal. Paris : Editions A. Pedone, 2012.

HANSON, Toby. Crime d'agression. Coalition pour la Cour pénale internationale. Disponible en: <http://www.iccnow.org/?mod=aggression\&lang=fr $>$. Accès le: 20 février 2016.

KELLER, Linda M. La fausse dichotomie entre paix et justice et la Cour pénale. Hague justice journal judiciaire de la Haye, v. 3, n. 1, p. 13-53, 2008.

KIRSH, Philippe. La Cour pénale internationale : de Rome à Kampala. Statut de Rome de la Cour pénale internationale. Paris: Pedone, 2012.

PASCUAL, Cristina Garcia. Norma Mundi La lucha por el derecho internacional. Madrid: Editorial Trotta, 2015.

POITEVI, Arnaud. Cour pénale internationale: les enquêtes et la latitude du Procureur. Droits fondamentaux, n. 4, p. 97-112, 2004.

SUR, Serge. Vers une Cour pénale internationale: la Convention de Rome entre les O.N.G. et le Conseil de sécurité. R.G.D.I.P, n.1, p. 29-45, 1999.

ZAPPALA, Salvatore. La justice pénale internationale. Paris: Editions Montchrestien, E.J.A., 2007. 
1 ZAPPALA, Salvatore. La justice pénale internationale. Paris: Editions Montchrestien, E.J.A., 2007.p. 146.

2 CASSESSE, Antonio. Los derechos humanos em el mundo contemporaneo. Barcelona: Ariel, 1993. p. 36.

3 FOFE DJOVIA MALEWA, Jean-Pierre. La Cour pénale internationale: institution nécessaire aux pays des grands lacs africains. Paris : L'Harmattan, 2006. p. 211.

4 FROUVILLE, Olivier de. Droit International pénal. Paris: Editions A. Pedone, 2012. p.17.

5 BOURGUIBA, Leïla. Cour pénale internationale, modèles de saisine et limites. Confluence méditerranée, Justice pénale et politique internationale. Paris, n. 64, p. 30, hiver 2007-2008.

6 PASCUAL, Cristina Garcia. Norma Mundi La lucha por el derecho internacional. Madrid: Editorial Trotta, 2015. p. 61.

7 ÇEHRELI, Ayse Sila. L'odyssée du XXe siècle: la naissance de la Cour pénale internationale. Synergies Turquie, n 2, 2009, pp. 113-122. p. 118.

8 BARCELO, Laurent. Aux origines de la Cour pénale internationale : le projet français de chambre criminelle internationale (hiver 1946 - printemps 1947). Guerres mondiales et conflits contemporains, n. 222, p. 103-109, 2/2006.

9 Article 16 de la Charte des Nations Unies: "Aucune enquête ni aucunes poursuites ne peuvent être engagées ni menées en vertu du présent Statut pendant les douze mois qui suivent la date à laquelle le Conseil de sécurité a fait une demande en ce sens à la Cour dans une résolution adoptée en vertu du Chapitre VII de la Charte des Nations Unies; la demande peut être renouvelée par le Conseil dans les mêmes conditions".

10 DULAIT, André. Rapport sur Le rôle du Conseil de sécurité sur le fonctionnement de la Cour pénale internationale. Sénat français, 1999. Disponible en: <http://www.senat.fr/ rap/r98-313/r98-3139.html>. Accès le: 15 février 2016.

11 Article 41 de la Charte des Nations Unies : "Le Conseil de sécurité peut décider quelles mesures n'impliquant pas l'emploi de la force armée doivent être prises pour donner effet à ses décisions, et peut inviter les Membres des Nations Unies à appliquer ces mesures. Celles-ci peuvent comprendre l'interruption complète ou partielle des relations économiques et des communications ferroviaires, maritimes, aériennes, postales, télégraphiques, radioélectriques et des autres moyens de communication, ainsi que la rupture des relations diplomatiques".

12 DULAIT, op. cit., 1999.

13 AMNESTY INTERNATIONAL. Bulletin d'information 228/2005. 2005. Disponible en: <http://www.amnesty.org/fr/library/asset/ASA57/004/2005/fr/62a24ca6-d4b7-11dd-8a23$\overline{\mathrm{d}} 58 \mathrm{a} 49 \mathrm{c} 0 \mathrm{~d} 652 / \mathrm{asa}$

$570042005 \mathrm{fr} . \mathrm{html}>$. Accès le: 15 février 2016.

14 KIRSH, Philippe. La Cour pénale international: de Rome à Kampala. Statut de Rome de la Cour pénale internationale. Paris: Pedone, 2012.

15 BOURGUIBA, op. cit., p. 30.

16 CENTRE D'ACTUALITES DE L'ONU. Libye: le Conseil de sécurité adopte des sanctions. Disponible en: <http://www.un.org/apps/newsFr/storyF.asp?NewsID $=24578 \& \mathrm{Cr}=\mathrm{Li}-$ bye\&Cr1>. Accès le: 20 février 2016.

17 CHIAVARIO, Mario. La justice pénale internationale entre passé et avenir. Paris: Editions DALLOZ, 2003. p. 113.

18 BOURGUIBA, op. cit., p. 29.

19 KIRSH, op. cit., p. 28.

20 Les Etats Unis, la Chine et la Russie.

21 La Résolution 1422 du Conseil de sécurité (d'abord adoptée en juillet 2002 puis renouvelée en juin 2003 sous la Résolution 1487) accorde l'immunité au personnel des Etats non parties de la CPI qui sont impliqués dans les missions établies ou autorisées des Nations unies pendant une durée renouvelable de douze mois. Malgré les efforts des Etats-Unis pour obtenir le renouvellement de cette résolution en 2004, ceux-ci ont retiré la résolution lorsqu'ils n'ont pas pu obtenir suffisamment de votes au Conseil de sécurité. Disponible en: $\leq$ http://www. iccnow.org/?mod=res1422\&lang=fr $\geq$. Accès le: 15 février 2016. 
22 Idem.

23 POITEVI, Arnaud. Cour pénale internationale: les enquêtes et la latitude du Procureur. Droits fondamentaux, n. 4, janvier - décembre 2004. p. 105.

24 KELLER, Linda M. La fausse dichotomie entre paix et justice et la Cour pénale. Hague justice journal judiciaire de la Haye, v. 3, n. 1, 2008.

25 POITEVI, op. cit., p. 105.

26 HANSON, Toby. Crime d'agression. Coalition pour la Cour pénale internationale. Disponible en: <http://www.iccnow.org/?mod=aggression\&lang=fr >. Accès le: 20 février 2016.

27 SUR, Serge. Vers une Cour pénale internationale: la Convention de Rome entre les O.N.G. et le Conseil de sécurité. R.G.D.I.P, n. 1, p. 44, 1999.

\title{
THE ROLE OF THE UNITED NATIONS SECURITY COUNCIL IN THE WORK OF THE INTERNATIONAL CRIMINAL COURT
}

\begin{abstract}
The Security Council is a political body concerned with the maintenance of peace and international security. The International Criminal Court, in its turn, is a subsidiary judicial body which aims at judging war crimes, genocide, aggression and crimes against humanity. Both bodies have a common denominator, which is the aim of maintaining peace and international security. This convergence of objectives was enshrined in the Rome Statute by providing the Security Council with the prerogative of referring cases to the Court. The intervention of the Security Council in the functioning of the Court may be beneficial to ensure Human Rights, however it may also represent an obstacle to the effective exercise of the jurisdiction of the Court. In this context, it would be interesting to analyze the constructive aspect of the relationship between the Court and the Security Council and the obstacle it may represent to the achievement of peace and international security. For this, qualitative methodology will be used. Documentary research, the study of jurisprudence and legislation will support the information relating to the subject.
\end{abstract}

Keywords: Security Council. International Criminal Court. Human Rights.

Submetido: $27 \mathrm{fev} .2016$

Aprovado: 13 maio 2016 